\title{
A CT-Based Radiomic Signature Can Be Prognostic for 10-Months Overall Survival in Metastatic Tumors Treated with Nivolumab: An Exploratory Study
}

\author{
Valentina D. A. Corino ${ }^{1, *}$, Marco Bologna ${ }^{1}$, Giuseppina Calareso ${ }^{2}$, Lisa Licitra ${ }^{3,4}{ }^{\text {, Mariagrazia Ghi }}{ }^{5}$, \\ Gaetana Rinaldi ${ }^{6}$, Francesco Caponigro ${ }^{7}$, Franco Morelli ${ }^{8}$, Mario Airoldi ${ }^{9}$, Giacomo Allegrini ${ }^{10}$, \\ Alessandra Cassano ${ }^{11}$, Daris Ferrari ${ }^{12}{ }^{\mathbb{D}}$, Aurora Mirabile ${ }^{13}{ }^{\mathbb{D}}$, Alicia Tosoni ${ }^{14}$, Danilo Galizia ${ }^{15}$, \\ Marco Merlano ${ }^{15,16} @$, Andrea Sponghini ${ }^{17}$, Gabriella Moretti ${ }^{18}$, Luca Mainardi ${ }^{1}$ and Paolo Bossi ${ }^{19}$
}

Citation: Corino, V.D.A.; Bologna, M.; Calareso, G.; Licitra, L.; Ghi, M.; Rinaldi, G.; Caponigro, F.; Morelli, F.; Airoldi, M.; Allegrini, G.; et al. A CT-Based Radiomic Signature Can Be Prognostic for 10-Months Overall Survival in Metastatic Tumors Treated with Nivolumab: An Exploratory Study. Diagnostics 2021, 11, 979. https://doi.org/10.3390/ diagnostics11060979

Academic Editor: Damiano Caruso

Received: 10 May 2021

Accepted: 25 May 2021

Published: 28 May 2021

Publisher's Note: MDPI stays neutral with regard to jurisdictional claims in published maps and institutional affiliations.

Copyright: (c) 2021 by the authors. Licensee MDPI, Basel, Switzerland. This article is an open access article distributed under the terms and conditions of the Creative Commons Attribution (CC BY) license (https:// creativecommons.org/licenses/by/ $4.0 /)$.
1 Department of Electronics, Information and Bioengineering (DEIB), Politecnico di Milano, 20133 Milan, Italy; marco.bologna@polimi.it (M.B.); luca.mainardi@polimi.it (L.M.)

2 Radiology Department, Fondazione IRCCS Istituto Nazionale dei Tumori di Milano, 20133 Milan, Italy; giuseppina.calareso@istitutotumori.mi.it

3 Head and Neck Medical Oncology Unit, Fondazione IRCCS Istituto Nazionale dei Tumori di Milano, University of Milan, 20133 Milan, Italy; lisa.licitra@istitutotumori.mi.it

4 Department of Oncology and Hemato-Oncology, University of Milan, 20122 Milan, Italy

5 Oncology 2 Unit, IRCCS Istituto Oncologico Veneto, 35128 Padua, Italy; mariagrazia.ghi@iov.veneto.it

6 Medical Oncology Unit, Policlinico P. Giaccone University Hospital, 90127 Palermo, Italy; taniarinaldi02@gmail.com

7 Head and Neck Medical and Experimental Oncology Unit, Istituto Nazionale Tumori, IRCCS Fondazione G. Pascale, 80131 Naples, Italy; f.caponigro@istitutotumori.na.it

8 Department of Oncology, IRCCS Casa Sollievo della Sofferenza, 71013 San Giovanni Rotondo, Italy; franco.morelli@gemellimolise.it

9 Medical Oncology 2 Unit, University Hospital “Città della Salute e della Scienza”, 10126 Turin, Italy; airoldim@yahoo.com

10 Azienda USL Toscana Nord Ovest, 56121 Tuscany, Italy; giacomo.allegrini@uslnordovest.toscana.it

11 Medical Oncology Unit, Policlinico Gemelli, 00168 Rome, Italy; alessandra.cassano@policlinicogemelli.it

12 Medical Oncology Unit, San Paolo Hospital, 20142 Milan, Italy; daris.ferrari@asst-santipaolocarlo.it

13 Medical Oncology Unit, San Raffaele Hospital, 20132 Segrate, Italy; mirabile.aurora@hsr.it

14 Medical Oncology Department, Azienda USL/IRCCS Istituto delle Scienze Neurologiche di Bologna, 40139 Bologna, Italy; a.tosoni@ausl.bologna.it

15 Multidisciplinary Outpatient Oncology Clinic, Candiolo Cancer Institute, FPO-IRCCS, 10060 Candiolo, Italy; danilo.galizia@ircc.it (D.G.); mcmerlano@gmail.com (M.M.)

16 Translational Research ARCO Foundation Cuneo, 12100 Cuneo, Italy

17 "Maggiore della Carità" University Hospital, 28100 Novara, Italy; asponghini@libero.it

18 GM Medical Oncology Unit, IRCCS Arcispedale S. Maria Nuova, 42123 Reggio Emilia, Italy;

Gabriella.Moretti@ausl.re.it

19 Medical Oncology, Department of Medical and Surgical Specialties, Radiological Sciences and Public Health University of Brescia, ASST-Spedali Civili, 25123 Brescia, Italy; paolo.bossi@unibs.it

* Correspondence: valentina.corino@polimi.it; Tel.: +0039-(02)-23993392

Abstract: Baseline clinical prognostic factors for recurrent and/or metastatic (RM) head and neck squamous cell carcinoma (HNSCC) treated with immunotherapy are lacking. CT-based radiomics may provide additional prognostic information. A total of 85 patients with RM-HNSCC were enrolled for this study. For each tumor, radiomic features were extracted from the segmentation of the largest tumor mass. A pipeline including different feature selection steps was used to train a radiomic signature prognostic for 10-month overall survival (OS). Features were selected based on their stability to geometrical transformation of the segmentation (intraclass correlation coefficient, ICC > 0.75) and their predictive power (area under the curve, AUC > 0.7). The predictive model was developed using the least absolute shrinkage and selection operator (LASSO) in combination with the support vector machine. The model was developed based on the first 68 enrolled patients and tested on the last 17 patients. Classification performance of the radiomic risk was evaluated accuracy and the AUC. The same metrics were computed for some baseline predictors used in clinical practice (volume of largest lesion, total tumor volume, number of tumor lesions, number of affected organs, 
performance status). The AUC in the test set was 0.67 , while accuracy was 0.82 . The performance of the radiomic score was higher than the one obtainable with the clinical variables (largest lesion volume: accuracy 0.59, AUC = 0.55; number of tumoral lesions: accuracy 0.71, AUC 0.36; number of affected organs: accuracy 0.47; AUC 0.42; total tumor volume: accuracy 0.59, AUC 0.53; performance status: accuracy $0.41, \mathrm{AUC}=0.47$ ). Radiomics may provide additional baseline prognostic value compared to the variables used in clinical practice.

Keywords: head and neck squamous cell carcinoma; radiomics; CT; overall survival

\section{Introduction}

Recurrent and/or metastatic (RM) head and neck squamous cell carcinoma (HNSCC) have a dismal prognosis in cases when salvage surgery or reirradiation could not be offered. Traditionally, chemotherapy combination and targeted antiEGFR agent cetuximab have been the mainstay of treatment for RM HNSCC, with overall response rate at around $36 \%$ and median overall survival of 10.1 months [1]. With the advent of immunotherapy, the landscape of treatment opportunities has changed in HNSCC, with the approval by regulatory agencies of immune checkpoint inhibitors for RM disease, namely nivolumab and pembrolizumab. These agents have been shown to improve overall survival (OS) in comparison to standard treatment, both in relapsing disease resistant to cisplatin chemotherapy and in settings in which the disease is still benefitting from cisplatin, as therapeutic combination or alone [2,3]. This survival benefit has been obtained mainly with the contribution of the subgroup of patients experiencing long-term survival: in fact, the 1 and 2-year OS has almost doubled in respect to standard comparator treatments [2,3]. Therefore, the next logical step in clinical research is the discovery of factors defining which group of patients would benefit mostly from immune checkpoint inhibitors. This would allow, on the one hand, maximization of their use in RM HNSCC and, on the other, the study of new treatment opportunities for patients without any foreseen advantage from immunotherapy alone. As of today, only the PD-L1 combined positive score (CPS) has been identified as a tool to select patients who could benefit from the use of pembrolizumab, alone or with chemotherapy, in platinum-sensitive HNSCC patients. However, a positive CPS is present in about $85 \%$ of patients and its specificity is quite limited, so underlining the need for more research in this field. Radiomic biomarkers have been studied as a tool to predict the benefit of immune checkpoint inhibitors, mainly in non-small-cell lung cancer and melanoma [4]. In previous studies, radiomics has been used as a surrogate for either PD-L1 positivity [5,6] or RECIST response to immunotherapy [7-12], while only a few studies have used radiomics to develop prognostic models for survival [13].

In the current paper, we present the results of radiomic analysis on pre-treatment images of patients receiving nivolumab for RM HNSCC patients to predict 10-month survival.

\section{Materials and Methods}

\subsection{Patient Population}

We considered patients enrolled in the "Nivactor" clinical trial, a phase IIIb trial with nivolumab $240 \mathrm{mg}$, in subjects with RM HNSCC resistant to platinum-based chemotherapy. The primary objective of the trial was to assess the incidence of high-grade (grade 3 or higher), treatment-related, selected adverse events (AE); secondary objectives were to characterize the outcome of all $\mathrm{AE}$, and to assess overall response rate, OS and Progression Free Survival (PFS). Main inclusion criteria of the trial were: histologically confirmed RM HNSCC (oral cavity, pharynx, larynx) not amenable to local therapy with curative intent; Eastern Cooperative Oncology Group (ECOG) performance status $\leq 2$; tumor progression or recurrence within 6 months of last dose of platinum therapy in the adjuvant (i.e., with radiation after surgery), primary (i.e., with radiation or prior to it, or to surgery as induction chemotherapy) or RM setting; measurable disease by CT or MRI per RECIST 1.1 criteria [14]. 
Baseline clinical data were considered in the analysis (age, sex, performance status, type of recurrence, number of lesions and site of metastasis), as well as biological characteristics (HPV status, as identified by p16 immunochemistry expression, and PD-L1 expression, as assessed by IHC $22 \mathrm{C} 3$ pharmDx)

For the current radiomic analysis (an ad-hoc of the clinical trial, not part of the protocol), we selected patients enrolled in the "Nivactor" project who met the following inclusion criteria: (1) availability of baseline CT imaging; (2) follow-up of at least 10 months. Out of 127 patients enrolled in the trial, a subset of 85 patients was included in our study. The main clinical and follow-up data for the selected patients are displayed in Table 1 . The 85 patients were divided into training and test sets based on the enrollment date, with the first 68 patients $(80 \%)$ assigned to the training set and the remaining $17(20 \%)$ assigned to the test set.

Table 1. Clinical data of patients involved in the study and best response to treatment. Numerical variables are expressed as median and inter-quartile range.

\begin{tabular}{|c|c|}
\hline \multicolumn{2}{|c|}{ Patients Clinical Data $(\mathrm{N}=85)$} \\
\hline Age at diagnosis (years) & $63(57-70)$ \\
\hline Sex & $\begin{array}{l}\text { Female: } 17(20 \%) \\
\text { Male: } 68(80 \%)\end{array}$ \\
\hline Performance status & $\begin{array}{l}\text { Status 0: } 27(32 \%) \\
\text { Status 1: } 55(65 \%) \\
\text { Status 2: } 3(3 \%)\end{array}$ \\
\hline PD-L1 expression & $\begin{array}{c}\text { Positive: } 29(34 \%) \\
\text { Negative: } 33(39 \%) \\
\text { Unknown: } 23(27 \%)\end{array}$ \\
\hline HPV status & $\begin{array}{c}\text { Positive: } 12(14 \%) \\
\text { Negative: } 12(14 \%) \\
\text { Unknown: } 61(72 \%)\end{array}$ \\
\hline Type of recurrence & $\begin{array}{c}\text { Local: } 9(11 \%) \\
\text { Regional: } 6(7 \%) \\
\text { Loco-regional: } 22(26 \%) \\
\text { Distant alone: } 19(22 \%) \\
\text { Distant + other: } 29(34 \%)\end{array}$ \\
\hline Number of lesions & $3(2-4)$ \\
\hline Number of affected organs & $2(1-3)$ \\
\hline RECIST response & $\begin{array}{c}\text { Progressive disease: } 55(65 \%) \\
\text { Stable disease: } 17(20 \%) \\
\text { Partial response: } 11(13 \%) \\
\text { Complete response: } 1(1 \%) \\
\text { Unknown: } 1(1 \%)\end{array}$ \\
\hline
\end{tabular}

\subsection{Image Acquisition}

Contrast-enhanced CT images were acquired for each of the 85 patients. Images were acquired using 25 different scanners (see Supplementary Materials Table S1, for the full list) and different image acquisition parameters such as pixel spacing, slice thickness, tube voltage and tube current. Details of the CT image acquisition parameter are reported in Supplementary Materials Table S2.

\subsection{Image Segmentation}

Each tumor mass was segmented by an expert radiologist (G.C.) with more than 10 years of experience. An example of segmented images is displayed in Supplementary Materials Figure S1. In case of multiple tumor masses, only the largest was segmented. 


\subsection{Image Preprocessing}

Image preprocessing was performed to reduce the imaging-related variability. First, a 3D Gaussian filter with a $3 \times 3 \times 3$ voxel kernel and $\sigma=0.5$ was used to denoise the images. Then, voxel size resampling to an isotropic resolution of $2 \mathrm{~mm}$ (as in [15]) was performed with B-spline interpolation.

\subsection{Radiomic Features Extraction}

The extraction of radiomic features was performed using Pyradiomics 3.0 [16]. A total of 536 features was extracted. The 536 features belonged to different categories: shape and size (14 features), first order statistics (18 features), textural (40 features), and wavelet (464 features). Textural features were computed using the grey level co-occurrence matrix (GLCM) and the grey level run length matrix (GLRLM). The full list of radiomic features is available in the Pyradiomics documentation [17]. A fixed-bin width histogram discretization ( 0.5 Hounsfield units per bin) was used prior to features extraction.

\subsection{Clinical Endpoint}

The clinical endpoint of interest for this exploratory ad-hoc radiomics study was overall survival (OS) at 10 months. OS was defined as the time (in days) between the beginning of treatment with nivolumab and the day of death or last contact. Moreover, we considered response to treatment according to RECIST 1.1 criteria [14].

\subsection{Radiomic Signature Training Pipeline}

Figure 1 shows the scheme, including all the steps for the training of the radiomic signature for survival classification.

The first postprocessing step was Z-score normalization, performed to ensure that the different features have similar ranges of values.

The normalized features underwent a series of feature selection steps that included the following: (1) selection of stable features; (2) selection of non-redundant features; (3) selection of features with high area under the curve (AUC) for the classification problem; (4) Least Absolute Shrinkage and Selection Operator (LASSO).

Only features that were stable to variations in the ROI were considered. Stability of features was assessed through small translations of the ROI ( $10 \%$ of the sizes of bounding box) as described in a previous study [18]. Stability was quantified using intra-class correlation coefficient (ICC) and features were considered stable if ICC was at least 0.75 [19].

Removal of redundant features was performed using pairwise correlation. When a pair of features had a Spearman correlation coefficient above 0.85 only one of the two was kept. In particular, only the one with lower mean correlation with all the other $n-2$ features was selected.

Among the non-redundant features, only those with high classification performance were selected for the next stage. The classification performance of the features was quantified using the AUC, and only those with AUC $>0.7$ were selected for the next step.

The final predictive model for 10-month survival prediction was developed using the least absolute shrinkage and selection operator (LASSO) in combination with support vector machine with cubic kernel [20]. LASSO is a type of model that depends on a tuning parameter $\lambda$, which controls the number of features used (the lower the $\lambda$, the higher the number of features). The optimal value for $\lambda$ was selected through internal cross-validation of the training set. The features selected by LASSO were given as input to a support vector machine classifier, thus deriving the radiomic signature. Patients with signature higher than threshold were classified as high-risk (HR) patients, while the others were classified as low-risk (LR) patients. The optimal threshold was estimated using the Receiver Operating Characteristic (ROC) curve of the training set. In particular, the threshold corresponding to the point of the curve closer to the optimal point of the ROC curve (sensitivity $100 \%$, false positive rate $0 \%$ ) was selected. Prior to the training of the classifier, data balancing 
was performed using the Synthetic Minority Oversampling Technique (SMOTE) [21], a technique used to artificially oversample a class.
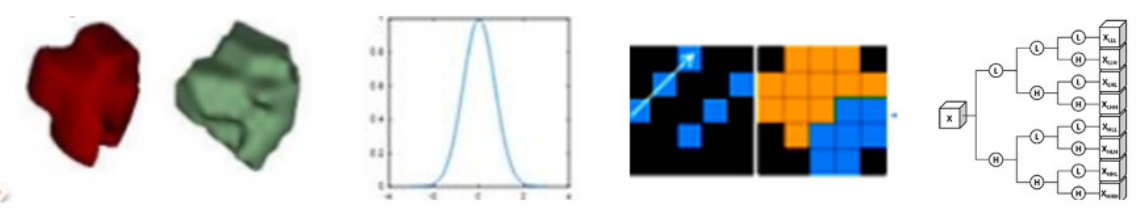

\section{Radiomic features}

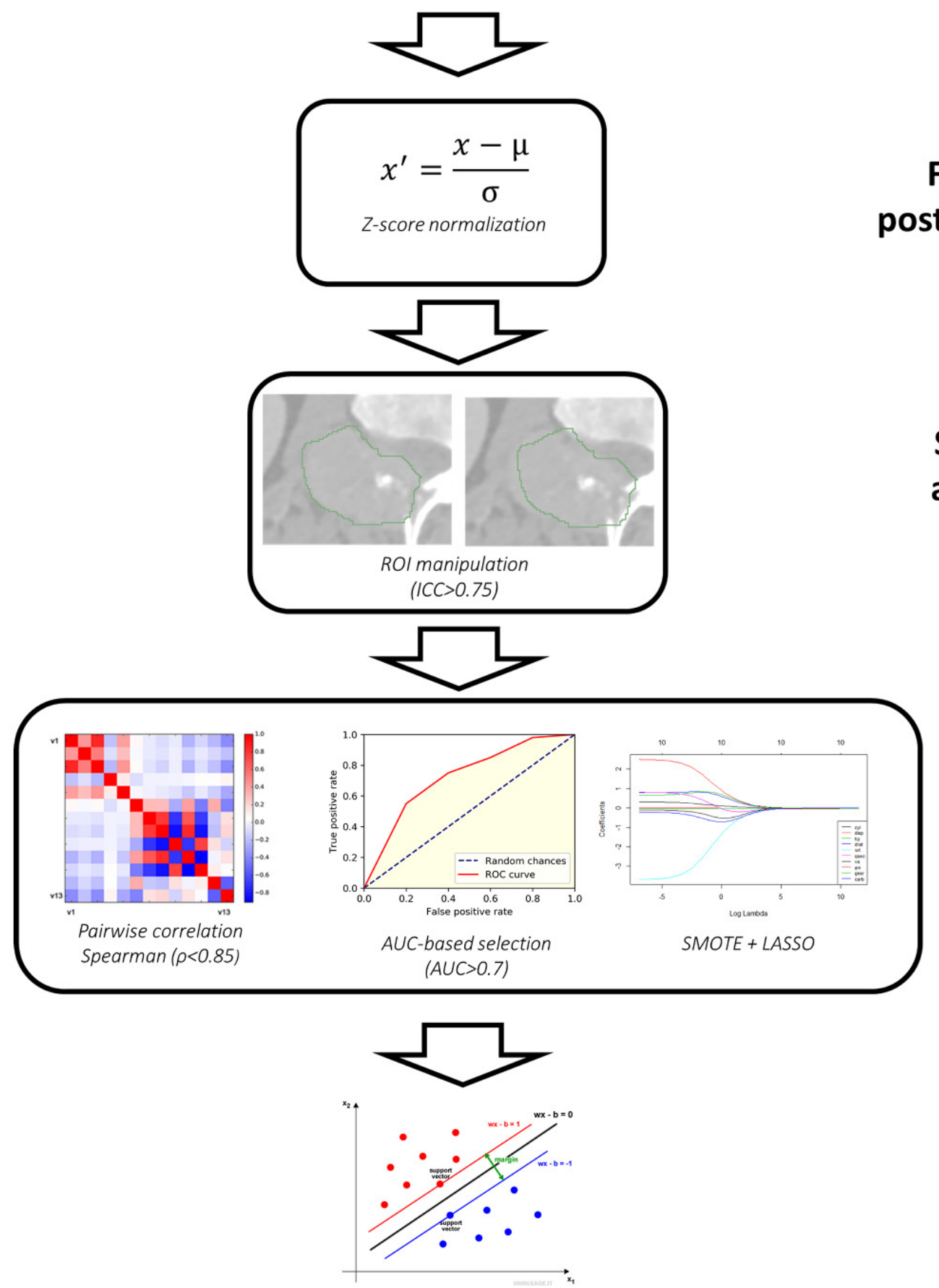

Stability analyses

Features postprocessing

Support Vector Machine + radiomic signature

Final results

Figure 1. Workflow for the training of the radiomic signature. 


\subsection{Radiomic Signature Validation}

To validate the performance of the trained model, we used the 17 patients in the test set. The images of the test set underwent Z-score normalization (using the mean and standard deviation estimated in the training set) and the same features selected in the training set were isolated.

The SVM model was applied to obtain the radiomic signature for the patients in the test set, and a threshold was applied to obtain the predicted class. The numeric signatures and the predicted classes are used to compute the confusion matrix and the ROC curve, which are used to compute the AUC, and the accuracy, sensitivity and specificity of the signature.

\subsection{Comparison of Radiomic Signature with Clinical Features}

To provide a reference for the performance of the radiomic signature, the classification performance of baseline predictors used in the clinical practice (volume of largest lesion, total tumor volume, number of tumor lesions, number of affected organs, performance status, presence of non-metastatic tumor) was evaluated. As for radiomic signature, AUC, accuracy, sensitivity and specificity of the model were used as quality metrics.

\subsection{Comparison with Volume}

Since it is known that signatures obtained from radiomic features may present high correlation with simple geometrical features such as volumes, and therefore become useless, the Spearman's correlation coefficient between ROI volume and the radiomic signature was computed.

\section{Results}

\subsection{Radiomic Signature Training and Validation}

The signature was composed of the following three features: original_shape_VoxelVolume, original-glrlm-RunLengthNonUniformity and waveletHLH-firstorder-Kurtosis.

Figure 2 shows the ROC curve and the confusion matrix for the radiomic-based classification, and the ROC curve of the radiomic signature in the test set. The binary radiomic classification reached an accuracy of $0.82(0.59-0.94)$, a sensitivity of $0.60(0.23-0.88)$ and a specificity of $0.92(0.65-0.99)$ with an AUC of $0.67(0.27-1)$ in the test set.

(A)

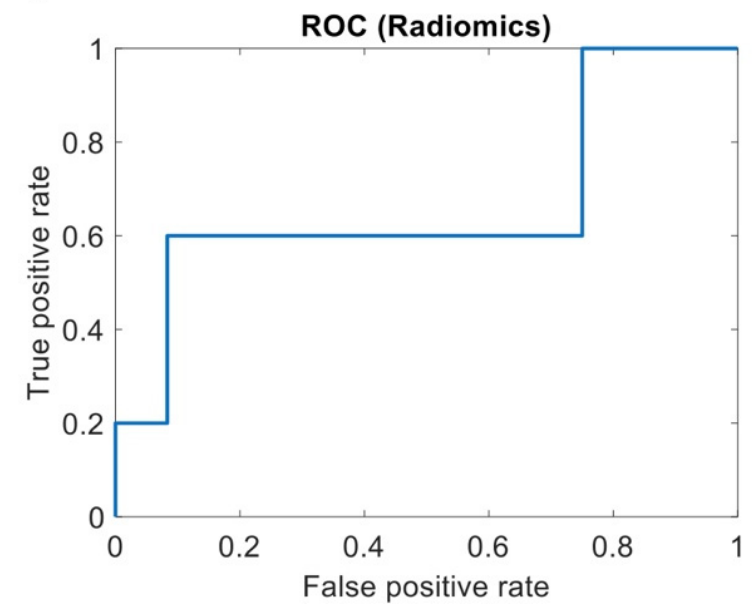

(B)

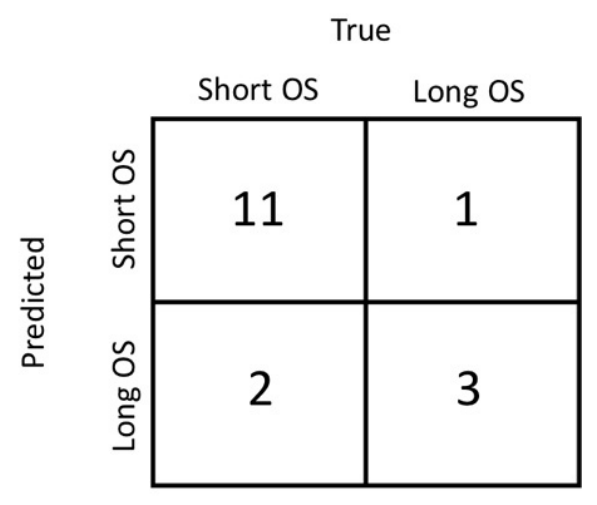

Figure 2. Performance of the radiomic signature on the test set. (A) Receiver operating characteristics (ROC) curve of the signature. (B) Confusion matrix. 


\subsection{Comparison with Clinical Features}

The ROC curves for the clinical variables, with the corresponding AUC, are displayed in Figure 3. Figure 4 displays the confusion matrices obtained using optimal threshold. Since for number of lesions and number of affected organs the ROC curves were strongly below 0.5 , the direction of the thresholding was inverted (patients with less than two regions or affected organs are considered long survivors). Table 2 sums up the quality metrics obtainable from the confusion matrices in Figure 4. It can be observed that for all clinical variables the AUC is close to 0.5, i.e., random classification, highlighting that the clinical variables are not able to predict the prognosis of patients with HNSCC.

(A)

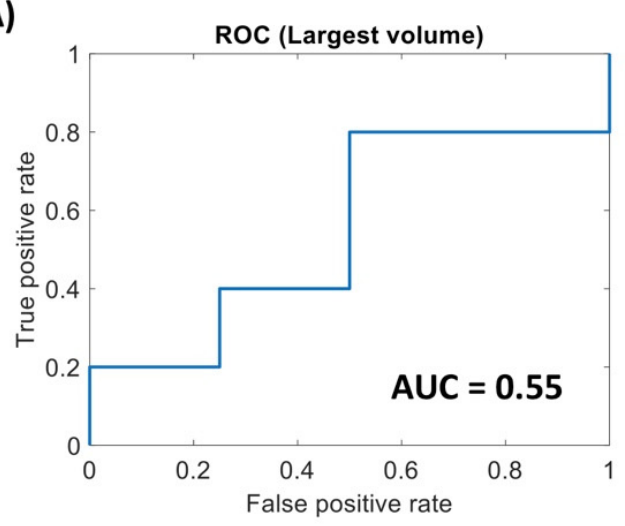

(C)

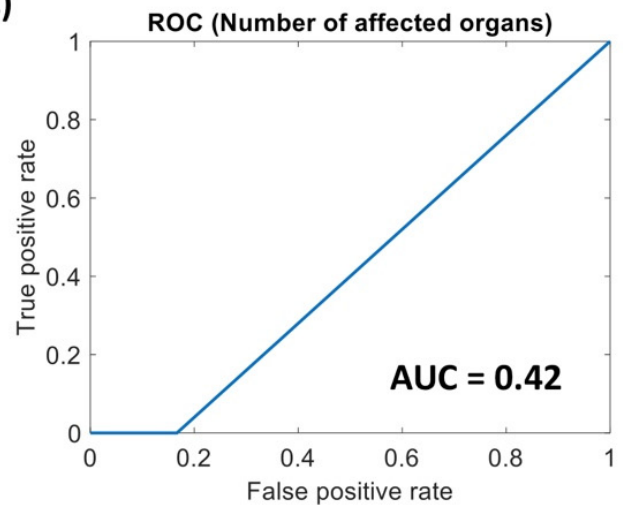

(E)

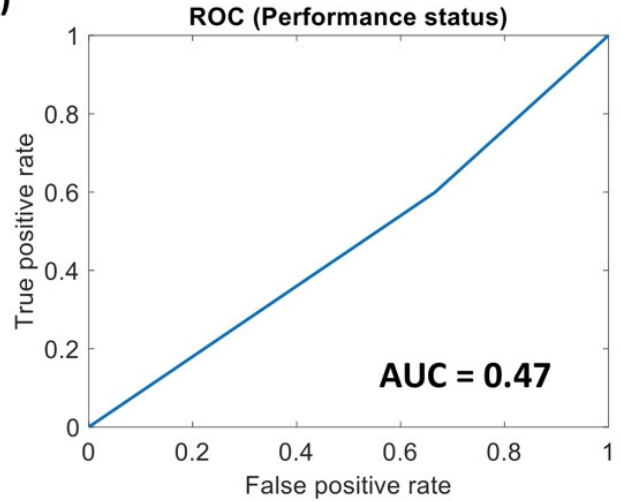

(B)

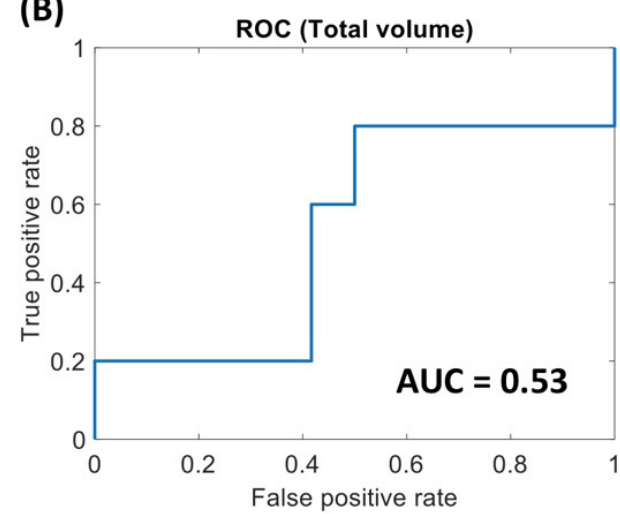

(D)

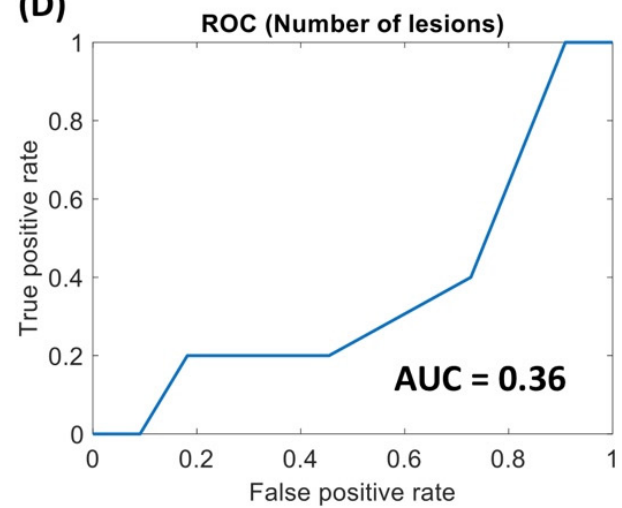

(F)

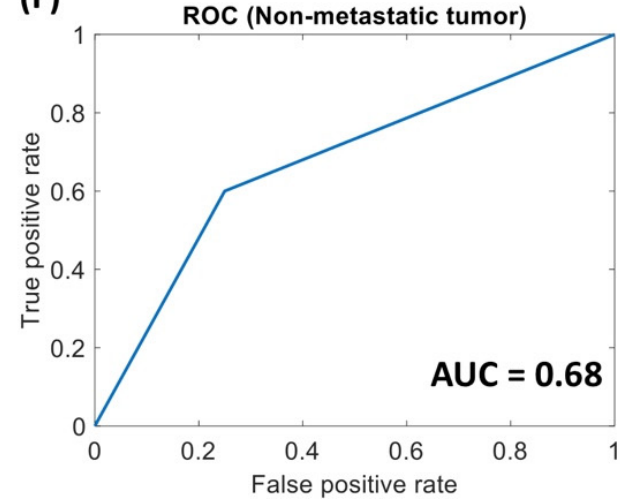

Figure 3. Receiver Operating Characteristics (ROC) curves for the clinical variables considered for the NIVACTOR project. (A) Volume of the largest Region Of Interest (ROI). (B) Total tumor volume. (C) Number of affected organs. (D) Number of lesions. (E) Performance status. (F) Non-metastatic status. 
(A)

\section{Largest volume}

Predicred

\begin{tabular}{|c|c|c|}
\hline & Short OS & Long OS \\
\hline $\begin{array}{l}\text { 乞 } \\
\text { to } \\
\text { ڤ }\end{array}$ & 6 & 6 \\
\hline $\begin{array}{l}\text { ก } \\
\text { on } \\
\text { ธ్ }\end{array}$ & 1 & 4 \\
\hline
\end{tabular}

(C) Number of affected organs

Predicted

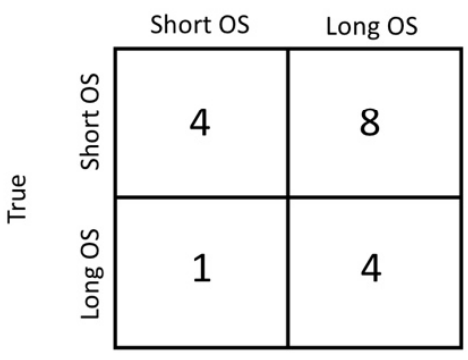

(E)

\section{Performance status}

Predicted

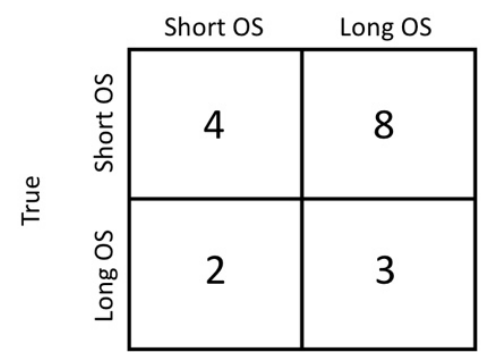

(B)

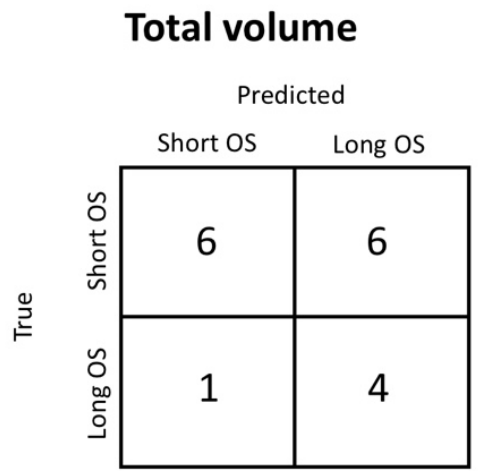

(D)

Number of lesions

Predicted

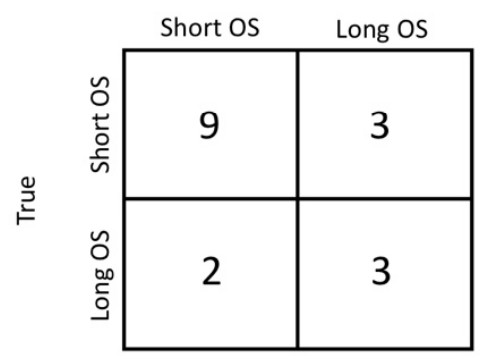

(F) Non-metastatic tumor

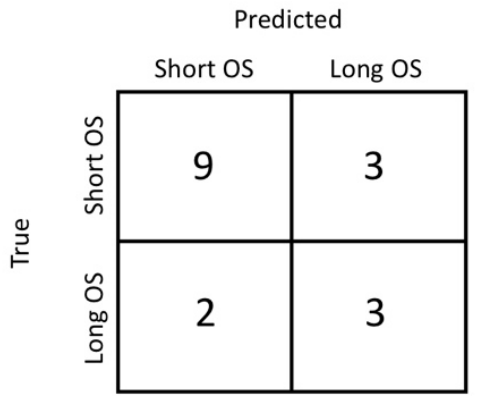

Figure 4. Confusion matrices for the clinical variables considered for the NIVACTOR project. (A) Volume of the largest Region Of Interest (ROI). (B) Total tumor volume. (C) Number of affected organs. (D) Number of lesions. (E) Performance status. (F) Non-metastatic status.

Table 2. Performance of the different variables in the prediction of long term survival (OS > 10 months).

\begin{tabular}{cccc}
\hline & \multicolumn{2}{c}{ Quality Metrics for Classification } & \\
\hline Variable & Accuracy & Sensitivity & Specificity \\
\hline Radiomics & $0.82(0.59-0.94)$ & $0.60(0.23-0.88)$ & $0.92(0.65-0.99)$ \\
Largest volume & $0.59(0.36-0.78)$ & $0.80(0.38-0.96)$ & $0.50(0.25-0.75)$ \\
Total volume & $0.59(0.36-0.78)$ & $0.80(0.38-0.96)$ & $0.50(0.25-0.75)$ \\
Number of lesions & $0.71(0.47-0.87)$ & $0.60(0.23-0.88)$ & $0.75(0.47-0.91)$ \\
Number of affected organs & $0.47(0.26-0.69)$ & $0.80(0.38-0.96)$ & $0.33(0.14-0.61)$ \\
Performance status & $0.41(0.22-0.64)$ & $0.60(0.23-0.88)$ & $0.33(0.14-0.61)$ \\
Non-metastatic tumor & $0.71(0.47-0.87)$ & $0.60(0.23-0.88)$ & $0.75(0.47-0.91)$ \\
\hline
\end{tabular}




\subsection{Comparison with Volume}

The scatterplot in Figure 5 displays the relationship between the volume of the ROI and the radiomic signature in the patients of the test set. The Spearman correction is weak and not significant (Spearman correlation coefficient $\rho=-0.36, p=0.15$ ). Therefore, tumor volume only explains part of the variability of the radiomic signature.

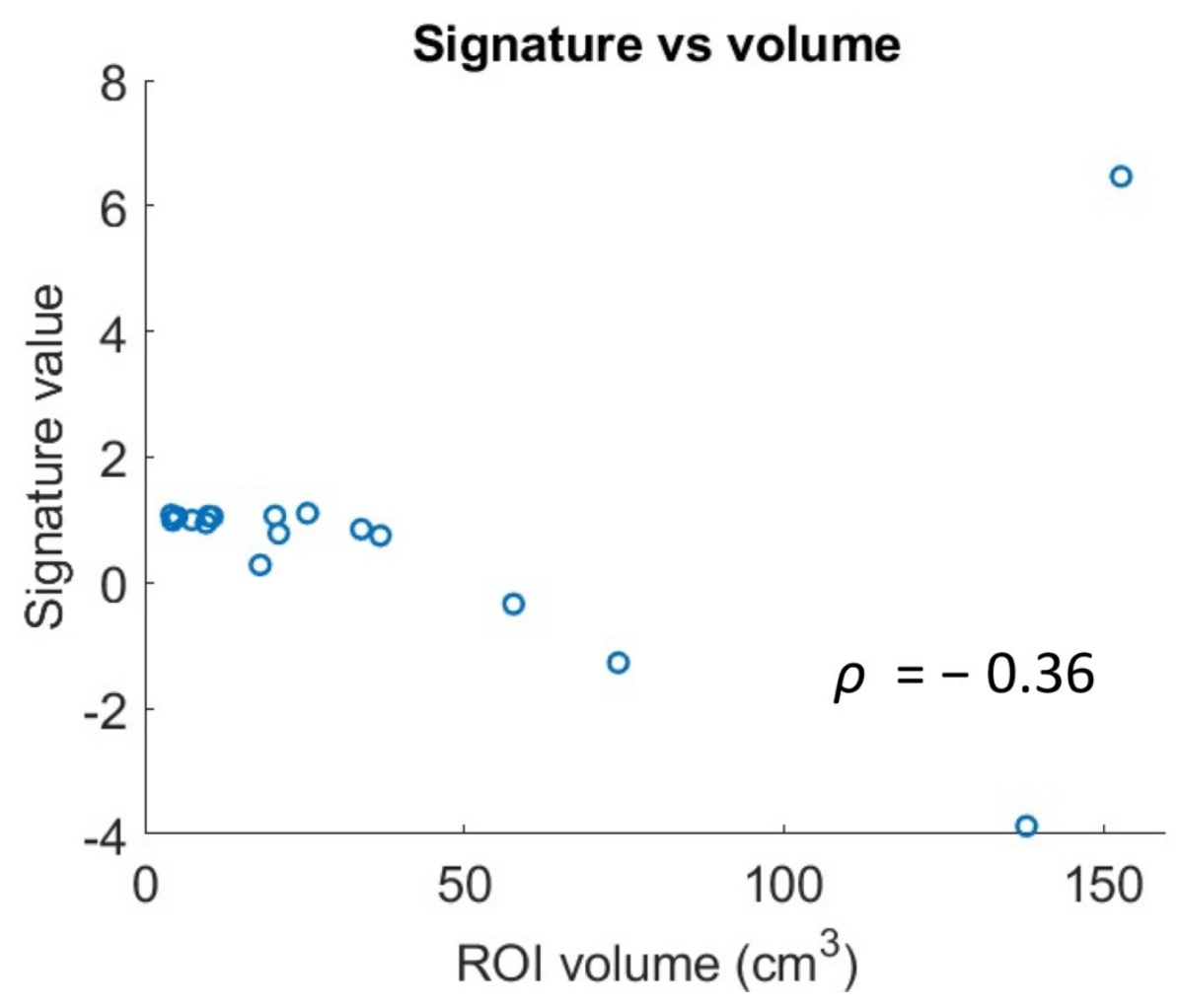

Figure 5. Scatterplot representing the correlation between the volume of the Region Of Interest (ROI) and the radiomic signature.

\section{Discussion}

Statement of principal findings:

The main findings of this proof-of-concept study are: (i) radiomic features can predict long (>10 months) survival from baseline imaging in patients with recurrent and/or metastatic, platinum-resistant HNSCC treated with nivolumab; (ii) the predictive power of the radiomic signature is higher than that of any other clinical variables acquired before immunotherapy treatment.

Meaning of the study: possible mechanisms and implications for clinicians:

The radiomic model was obtained after proper feature selection by using a support vector machine with a cubic kernel. The three features that were used to train the model were the following: original-shape-VoxelVolume, original-glrlm-RunLengthNonUniformity and waveletHLH-firstorder-Kurtosis.

A low value of original-glrlm-RunLengthNonUniformity is associated with runs that are equally distributed along run lengths [22], so a higher value of the feature results in runs of a particular length that are more frequent than others. To get a better interpretation of the features it may be considered that, in this dataset, original-glrlm-RunLengthNonUniformity is positively correlated with original-glrlm-GreyLevelNonUniformity (Spearman's correlation coefficient $0.91, p=1.13 \times 10^{-27}$ ), which has been used in the past to describe intra-tumoral heterogeneity [23]. The waveletHLH transform highlights the high frequencies (high pass filter) in the $\mathrm{x}$ and $\mathrm{z}$ direction and the low frequencies (low pass filter) in the $y$ direction and kurtosis measures the peakedness of the intensity distribution [22]; therefore, a higher waveletHLH-firstorder-Kurtosis indicates a higher peakedness of the 
grey values in the waveletHLH of the original image. Such a feature is also positively correlated with original-glrlm-GreyLevelNonUniformity (Spearman's correlation coefficient $0.57, p=1.06 \times 10^{-8}$ ), so the higher the feature, the higher the intra-tumor heterogeneity. Moreover, kurtosis has been previously used in in prognostic models for OS [13,24]. Finally, tumor volume has been shown to be a prognostic factor for OS [25] and so it is reasonable that tumor volume is an important feature in determining survival class (long/short). However, the results show that the volume of the largest ROI alone is not enough to obtain good results on the test set. The radiomic score contains an added value compared to volume alone, which is evident from the better classification results and by the low correlation of tumor volume and the radiomic score.

It is possible to see that the radiomic score on which the classification is based is the feature with the best predictive performance among those obtainable at baseline (AUC $=0.67$, accuracy 0.82). Of the clinical features, the number of lesions has an AUC much lower than 0.5 (AUC $=0.36$ ), and therefore using an inverted threshold (in this case $n \leq 2$ ) also provides good prognostic accuracy, with the same sensitivity but lower specificity compared to the radiomic features. The number of affected organs has an AUC $=0.58$, but the selected threshold leads to a low accuracy (0.41). The other three clinical variables had low AUC and accuracy.

Strengths and weaknesses of the study:

The preliminary results seem to suggest that radiomics may provide a stronger tool for prediction of long-term survival of patients treated with nivolumab using only information available at baseline (i.e., medical imaging). The prediction of the benefit of immune checkpoint inhibitors in HNSCC appears to be challenging, as clinical features seem not to be predictive. Therefore, this study, even if only preliminary, may be a first step in better understanding response to immunotherapy and thus prognosis of patients with HNSCC.

A limitation of the study is the limited size of the population, which makes the study only a proof-of-concept, requiring further validation sets.

Strengths and weaknesses in relation to other studies, discussing particularly any differences in results:

This was not the first study in which radiomics has been applied to define the prognosis of patients treated with immunotherapy [5,7-9,12-26], even though most of the studies refer to lung cancers $[5,7,8,12]$. In most of these studies, the developed models were referred to RECIST response, but in [12] the predictive power for long term OS (15 months) was investigated starting from PET features. Our study investigates the performance of CT imaging, which is more frequently used and could provide additional information.

In this regard, it should be underlined that immunotherapy in cancer patients obtains its advantages mostly by increasing long-term survival; this is particularly evident in HNSCC, where the use of immune checkpoint inhibitors formed a plateau in the survival curves, which has not been previously reached with chemotherapy and targeted agents. Moreover, immunotherapy could prime the tumoral microenvironment for response to further chemotherapy lines, and consequently obtain increased survival [27].

Unanswered questions and future research:

So far, we lack clinical and biological factors able to predict the benefit of immune checkpoint inhibitors in HNSCC, except for performance status and PD-L1 status, even if the role of the latter is confirmed only in patients treated with pembrolizumab, with or without chemotherapy, in platinum-sensitive diseases. Therefore, when validated in larger series, CT-radiomics can provide an additional source of information that may help the prognostic performance for OS. Moreover, possible future developments of radiomics could include the development of a non-invasive characterization of tumor microenvironment [28] to provide better tumor characterization and exploit the information in predictive and prognostic models. 


\section{Conclusions}

In this proof-of-concept study radiomic features were able to predict long/short survival (>10 months) at baseline. The predictive power of the radiomic features was found to be higher than that of any other variables acquired before immunotherapy treatment. Although preliminary, the results of this study may be a first step in a path that leads to the use of CT-radiomics in clinical practice.

Supplementary Materials: The following are available online at https://www.mdpi.com/article/ 10.3390/diagnostics11060979/s1. Table S1, Distribution of the different scanners used in the study. Table S2, Image acquisition parameters for the computed tomography (CT) images used in the studies. Numeric values are expressed as median and inter-quartile ranges. Figure S1, Example of computed tomography image with the segmented tumor.

Author Contributions: V.D.A.C., radiomic features extraction, predictive model training and validation, manuscript writing; M.B., radiomic features extraction, predictive model training and validation, manuscript writing; G.C.: image segmentation, image data collection and cleaning, manuscript revision; L.L.: data collection, manuscript revision; M.G.: data collection, manuscript revision; G.R.: data collection, manuscript revision; F.C.: data collection, manuscript revision; F.M.: data collection, manuscript revision; M.A.: data collection, manuscript revision; G.A.: data collection, manuscript revision; A.C.: data collection, manuscript revision; D.F.: data collection, manuscript revision; A.M.: data collection, manuscript revision; A.T.: data collection, manuscript revision; D.G.: data collection, manuscript revision; M.M.: data collection, manuscript revision; A.S.: data collection, manuscript revision; G.M.: data collection, manuscript revision; L.M.: supervision, manuscript revision; P.B.: clinical data collection, supervision, manuscript revision. All authors have read and agreed to the published version of the manuscript.

Funding: This paper has been possible thanks to the support of AIRC IG 2018 Grant ID 21740 to PB. Nivolumab has been provided for free by Bristol Meyer Squibb.

Institutional Review Board Statement: The study was conducted according to the guidelines of the Declaration of Helsinki and approved by the Institutional Review Board (or Ethics Committee) of Fondazione IRCCS Istituto Nazionale dei Tumori, Milan (protocol Eudract code 2017-000562-30, approved on 08-09-2017).

Informed Consent Statement: Informed consent was obtained from all subjects involved in the study.

Data Availability Statement: The data presented in this study are available on request from the corresponding author. The data are not publicly available due to restrictions due to privacy issues.

Conflicts of Interest: The authors declare no conflict of interest.

\section{References}

1. Vermorken, J.B.; Mesia, R.; Rivera, F.; Remenar, E.; Kawecki, A.; Rottey, S.; Erfan, J.; Zabolotnyy, D.; Kienzer, H.-R.; Cupissol, D.; et al. Platinum-Based Chemotherapy plus Cetuximab in Head and Neck Cancer. N. Engl. J. Med. 2008, 359, 1116-1127. [CrossRef]

2. Ferris, R.L.; Fayette, J.; Guigay, J.; Dimitrios, A.; Licitra, L.; Harrington, K.J.; Kasper, S.; Vokes, E.E.; Worden, F.; Saba, N.F.; et al. Nivolumab vs investigator's choice in recurrent or metastatic squamous cell carcinoma of the head and neck: 2-year long-term survival update of CheckMate 141 with analyses by tumor PD-L1 expression. Oral Oncol. 2018, 81, 45-51. [CrossRef]

3. Burtness, B.; Harrington, K.J.; Greil, R.; Soulières, D.; Tahara, M.; de Castro, G.; Psyrri, A.; Basté, N.; Neupane, P.; Bratland, Å.; et al. Pembrolizumab alone or with chemotherapy versus cetuximab with chemotherapy for recurrent or metastatic squamous cell carcinoma of the head and neck (KEYNOTE-048): A randomised, open-label, phase 3 study. Lancet 2019, 394, 1915-1928. [CrossRef]

4. Zhang, C.; de A. F. Fonseca, L.; Shi, Z.; Zhu, C.; Dekker, A.; Bermejo, I.; Wee, L. Systematic review of radiomic biomarkers for predicting immune checkpoint inhibitor treatment outcomes. Methods 2020, 188, 61-72. [CrossRef] [PubMed]

5. Sun, R.; Limkin, E.J; Vakalopoulou, M.; Dercle, L.; Champiat, S.; Han, S.R.; Verlingue, L.; Brandao, D.; Lancia, A.; Ammari, S.; et al. A radiomics approach to assess tumour-infiltrating CD8 cells and response to anti-PD-1 or anti-PD-L1 immunotherapy: An imaging biomarker, retrospective multicohort study. Lancet Oncol. 2018, 19, 1180-1191. [CrossRef]

6. Yoon, J.; Suh, Y.J.; Han, K.; Cho, H.; Lee, H.J.; Hur, J.; Choi, B.W. Utility of CT radiomics for prediction of PD-L1 expression in advanced lung adenocarcinomas. Thorac. Cancer 2020, 11, 993-1004. [CrossRef] 
7. Khorrami, M.; Prasanna, P.; Gupta, A.; Patil, P.; Velu, P.D.; Thawani, R.; Corredor, G.; Alilou, M.; Bera, K.; Fu, P.; et al. Changes in CT radiomic features associated with lymphocyte distribution predict overall survival and response to immunotherapy in non-small cell lung cancer. Cancer Immunol. Res. 2020, 8, 108-119. [CrossRef]

8. Polverari, G.; Ceci, F.; Bertaglia, V.; Reale, M.L.; Rampado, O.; Gallio, E.; Passera, R.; Liberini, V.; Scapoli, P.; Arena, V.; et al. 18 F-FDG pet parameters and radiomics features analysis in advanced nsclc treated with immunotherapy as predictors of therapy response and survival. Cancers 2020, 12, 1163. [CrossRef] [PubMed]

9. Ji, Z.; Cui, Y.; Peng, Z.; Gong, J.; Zhu, H.T.; Zhang, X.; Li, J.; Lu, M.; Lu, Z.; Shen, L.; et al. Use of radiomics to predict response to immunotherapy of malignant tumors of the digestive system. Med. Sci. Monit. 2020, 26, e924671-1-e924671-9.

10. Tunali, I.; Gray, J.E.; Qi, J.; Abdalah, M.; Jeong, D.K.; Guvenis, A.; Gillies, R.J.; Schabath, M.B. Novel clinical and radiomic predictors of rapid disease progression phenotypes among lung cancer patients treated with immunotherapy: An early report. Lung Cancer 2019, 129, 75-79. [CrossRef]

11. Trebeschi, S.; Drago, S.G.; Birkbak, N.J.; Kurilova, I.; Călin, A.M.; Delli Pizzi, A.; Lalezari, F.; Lambregts, D.M.J.; Rohaan, M.W.; Parmar, C.; et al. Predicting response to cancer immunotherapy using noninvasive radiomic biomarkers. Ann. Oncol. 2019, 30, 998-1004. [CrossRef]

12. Valentinuzzi, D.; Vrankar, M.; Boc, N.; Ahac, V.; Zupancic, Z.; Unk, M.; Skalic, K.; Zagar, I.; Studen, A.; Simoncic, U.; et al. FDG PET immunotherapy radiomics signature (iRADIOMICS) predicts response of non-small-cell lung cancer patients treated with pembrolizumab. Radiol. Oncol. 2020, 54, 285-294. [CrossRef]

13. Schraag, A.; Klumpp, B.; Afat, S.; Gatidis, S.; Nikolaou, K.; Eigentler, T.K.; Othman, A.E. Baseline clinical and imaging predictors of treatment response and overall survival of patients with metastatic melanoma undergoing immunotherapy. Eur. J. Radiol. 2019, 121, 108688. [CrossRef] [PubMed]

14. Eisenhauer, E.A.; Therasse, P.; Bogaerts, J.; Schwartz, L.H.; Sargent, D.; Ford, R.; Dancey, J.; Arbuck, S.; Gwyther, S.; Mooney, M.; et al. New response evaluation criteria in solid tumours: Revised RECIST guideline (version 1.1). Eur. J. Cancer 2009, 45, 228-247. [CrossRef] [PubMed]

15. Leijenaar, R.T.; Bogowicz, M.; Jochems, A.; Hoebers, F.J.; Wesseling, F.W.; Huang, S.H.; Chan, B.; Waldron, J.N.; O'Sullivan, B.; Rietveld, D.; et al. Development and validation of a radiomic signature to predict HPV (p16) status from standard CT imaging: A multicenter study. Br. J. Radiol. 2018, 91, 20170498. [CrossRef] [PubMed]

16. van Griethuysen, J.J.M.; Fedorov, A.; Parmar, C.; Hosny, A.; Aucoin, N.; Narayan, V.; Beets-Tan, R.G.H.; Fillion-Robin, J.-C.; Pieper, S.; Aerts, H.J.W.L. Computational Radiomics System to Decode the Radiographic Phenotype. Cancer Res. 2017, 77, e104-e107. [CrossRef] [PubMed]

17. Pyradiomics Features Description. Available online: https:// pyradiomics.readthedocs.io/en/2.1.0/features.html (accessed on 4 October 2020).

18. Bologna, M.; Corino, V.D.A.; Montin, E.; Messina, A.; Calareso, G.; Greco, G.; Sdao, S. Assessment of Stability and Discrimination Capacity of Radiomic Features on Apparent Diffusion Coefficient Images. J. Digit. Imaging 2018, 31, 879-894. [CrossRef] [PubMed]

19. Koo, T.K.; Li, M.Y. A Guideline of Selecting and Reporting Intraclass Correlation Coefficients for Reliability Research. J. Chiropr. Med. 2016, 15, 155-163. [CrossRef]

20. James, G.; Witten, D.; Hastie, T.; Tibshirani, R. An Introduction to Statistical Learning-With Applications in R; Springer: Berlin/Heidelberg, Germany, 2013; ISBN 9781461471370.

21. Chawla, N.V.; Bowyer, K.W.; Hall, L.O.; Kegelmeyer, W.P. SMOTE: Synthetic Minority Over-sampling Technique. J. Artif. Intell. Res. 2002, 16, 321-357. [CrossRef]

22. Zwanenburg, A.; Vallières, M.; Abdalah, M.A.; Aerts, Hugo J. W. L.; Apte, A.; Ashrafinia, S.; Bakas, S.; Beukinga, R.J.; Boellaard, R.; Bogowicz, M.; et al. Image biomarker standardisation initiative. Radiology 2020, 328-338. [CrossRef]

23. Aerts, H.J.W.L.; Velazquez, E.R.; Leijenaar, R.T.H.; Parmar, C.; Grossmann, P.; Cavalho, S.; Bussink, J.; Monshouwer, R.; HaibeKains, B.; Rietveld, D.; et al. Decoding tumour phenotype by noninvasive imaging using a quantitative radiomics approach. Nat. Commun. 2014, 5, 4006. [CrossRef]

24. Tang, C.; Hobbs, B.; Amer, A.; Li, X.; Behrens, C.; Canales, J.R.; Cuentas, E.P.; Villalobos, P.; Fried, D.; Chang, J.Y.; et al. Development of an Immune-Pathology Informed Radiomics Model for Non-Small Cell Lung Cancer. Sci. Rep. 2018, 8, 1-9. [CrossRef]

25. Welch, M.L.; McIntosh, C.; Haibe-Kains, B.; Milosevic, M.F.; Wee, L.; Dekker, A.; Huang, S.H.; Purdie, T.G.; O’Sullivan, B.; Aerts, H.J.W.L.; et al. Vulnerabilities of radiomic signature development: The need for safeguards. Radiother. Oncol. 2019, 130, 2-9. [CrossRef] [PubMed]

26. Dercle, L.; Fronheiser, M.; Lu, L.; Du, S.; Hayes, W.; Leung, D.K.; Roy, A.; Wilkerson, J.; Guo, P.; Fojo, A.T.; et al. Identification of non-small cell lung cancer sensitive to systemic cancer therapies using radiomics. Clin. Cancer Res. 2020, 26, $2151-2162$. [CrossRef] [PubMed]

27. Saleh, K.; Daste, A.; Martin, N.; Pons-Tostivint, E.; Auperin, A.; Herrera Gómez, R.G.; Baste Rotllan, N.; Bidault, F.; Guigay, J.; Le Tourneau, C.; et al. Response to salvage chemotherapy after progression on immune checkpoint inhibitors in patients with squamous cell carcinoma of the head and neck. Eur. J. Cancer 2019, 121, 123-129. [CrossRef]

28. Yoon, H.J.; Kang, J.; Park, H.; Sohn, I.; Lee, S.H.; Lee, H.Y. Deciphering the tumor microenvironment through radiomics in non-small cell lung cancer: Correlation with immune profiles. PLoS ONE 2020, 15, e0231227. [CrossRef] [PubMed] 